\title{
Development of Motor Networks in Zebrafish Embryos
}

\author{
LOUIS SAINT-AMANT
}

\begin{abstract}
General mechanisms of motor network development have often been examined in the spinal cord because of its relative simplicity when compared to higher parts of the brain. Indeed, most of our current understanding of motor pattern generation comes from work in the lower vertebrate spinal cord. Nevertheless, very little is known about the initial stages of motor network formation and the interplay between genes and electrical activity. Recent research has led to the establishment of the zebrafish as a key model system to study the genetics of neural development. The spinal cord of zebrafish is amenable to optical and electrophysiological analysis of neuronal activity even at the earliest embryonic stages when the network is immature. The combination of physiology and genetics in the same animal model should lead to insights into the basic mechanisms of motor circuit formation. This paper reviews recent work on the development of zebrafish motor activity and discusses them in the context of the current knowledge of embryonic and larval zebrafish spinal cord morphology and physiology.
\end{abstract}

\section{INTRODUCTION}

$\boldsymbol{S}_{\mathrm{r}}^{\mathrm{p}}$ PONTANEOUS ELECTRICAL ACTIVITY is a common feature of developing neuronal networks. ${ }^{1-3}$ This activity is thought to refine synaptic connections by providing cues for appropriate neural wiring. ${ }^{4-6}$ Spontaneous activity has also been proposed to affect cell fate determination, ${ }^{7}$ and more recently, the control of neurotransmitter expression in embryonic neurons. ${ }^{8,9}$ Developing vertebrate embryos, either in the egg or womb, undergo a period of spontaneous motor activity that is generated by the developing motor network. This spontaneous motor activity has been shown in many animal models to emanate from a central pattern generator that is independent of sensory inputs. ${ }^{10-17}$ Perturbing this early pattern of spontaneous activity has recently been shown to affect motor axon guidance in the chick embryo $^{18}$ and disturb the assembly of spinal motor networks in the mouse. ${ }^{19}$

Most of what we know about the vertebrate central pattern generator (CPG) for locomotion has been learned from decades of work studying swimming in Xenopus larvae and lamprey. ${ }^{20-23}$ The limited genetic and molecular tools for these animal models have restricted our understanding of motor circuit formation to the cellular level. The zebrafish has recently emerged as an important model to study the genetics of neural circuit development. ${ }^{24,25}$ In the past decade, mutagenesis screens have yielded mutants affecting the central nervous system. ${ }^{26,27}$ In addition, electrophysiological and imaging techniques have been developed to study the electrical activity of zebrafish embryos and larvae in vivo. ${ }^{28-40}$ Previous reviews have covered the early steps in the development of the spinal cord in zebrafish such as neurogenesis, ${ }^{41,42}$ axon guidance, ${ }^{43-45}$ and the emergence of motor circuits. ${ }^{42,46,47}$ This review will focus on what is currently known about the emergence and maturation of zebrafish motor behaviors and the progressive changes in the neuronal cir-

University of Michigan, Ann Arbor, Michigan. 
cuits that are necessary to produce these activity patterns.

\section{SPONTANEOUS MOTILITY AND EARLY SPINAL CORD MORPHOLOGY}

Zebrafish embryos show their first motor activity at $17 \mathrm{~h}$ postfertilization (hpf). ${ }^{48,49}$ This immature motor behavior consists of spontaneous repeating, alternating coils of the tail that persist over the course of several hours. The frequency of these coils in dechorionated embryos peaks at $1 \mathrm{~Hz}$ at $19 \mathrm{hpf}$ and slowly decreases to $0.1 \mathrm{~Hz}$ by $26 \mathrm{hpf}$. The spontaneous coils were shown to be neural in origin by abolishing the coils with injections of nicotinic acetylcholine receptor blockers. ${ }^{49,50}$ The substrate essential for the appearance of spontaneous coils is located completely within the spinal cord, as lesions that remove all brain structures above the spinal cord do not affect the frequency or strength of the coils. ${ }^{49}$

Zebrafish spontaneous motor activity appears at a developmental time when the spinal cord is immature. Indeed, the first postmitotic neurons in the zebrafish spinal cord extend their axons at $15 \mathrm{hpf}$, a mere $2 \mathrm{~h}$ before the appearance of motor behavior ${ }^{51-53}$ and even by 21 hpf there are only six types of neurons bearing axons (Fig. 1A). The spinal cord of a zebrafish embryo is divided into repeating segments, or somites. At the dorsal most edge of each segment we find two-three Rohon-Beard sensory neurons, these cells are the first spinal cord neurons to extend axons in the dorsolateral fasciculus (DLF) of the spinal cord. Dorsolateral ascending (DoLA) interneurons have one-two somata per segment, are located slightly more ventrally than Rohon-Beard neurons and project their axons rostrally in the DLF. Commissural primary ascending (CoPA) interneurons have one-two somata per somite that are located dorsally and project axons first ventrally before crossing to the other side of the spinal cord and projecting rostrally in the DLF.

There are only two types of interneurons that project descending axons ipsilaterally along several somites as early as $17 \mathrm{hpf}$. First, the IC interneurons (ipsilateral caudal axon) are a population of early born neurons, with numbers ranging from one to two per segment, that span the hindbrain/spinal cord border but are not present after the sixth somite. ${ }^{54,55}$ The other descending interneuron type is the ventral lateral descending (VeLD). VeLD cell bodies are located throughout the spinal cord with about two cells per segment. ${ }^{51,52}$ Before $26 \mathrm{hpf}$ there are only three motoneurons per side of each segment: the CaP (caudal primary), MiP (middle primary), and RoP (rostral primary) motoneurons. ${ }^{56-58}$ The first contact between motoneurons and muscle is attained at $17 \mathrm{hpf}$. These initial contacts are coincident with the appearance of the spontaneous behavior, suggesting that the spinal circuitry may be active slightly before motoneuron-muscle contact.

These morphological observations suggest that a maximum of six cell types located wholly within the immature embryonic spinal cord are responsible for the appearance of spontaneous motor activity. These low numbers of neurons suggest that a simple neuronal circuit underlies the earliest form of behavior in zebrafish.

\section{A GAP JUNCTION-MEDIATED IMMATURE SPINAL NETWORK}

The strong rhythmic pattern of behavior observed in the embryo suggests that the electrical activity in the spinal cord should also be rhythmic in nature, although the type of electrical activity and method of propagation are unclear. In order to visualize the source of these motor patterns, in vivo whole-cell patch clamp recordings of spinal neuron activity were obtained from paralyzed embryos. ${ }^{30,38}$ Cell attached recordings of spiking activity were first obtained from motoneurons. ${ }^{59}$ The activity observed in the cell-attached configuration consists of repetitive patterns of one or more spikes (Fig. 1B). As the embryos mature, the bursts become less frequent but contain more spikes. As would be expected for a centrally generated and sensory independent behavior, the average frequency of bursting in motoneurons from paralyzed embryos matches the average frequency of the spontaneous coiling behavior observed at all ages. ${ }^{59}$ 


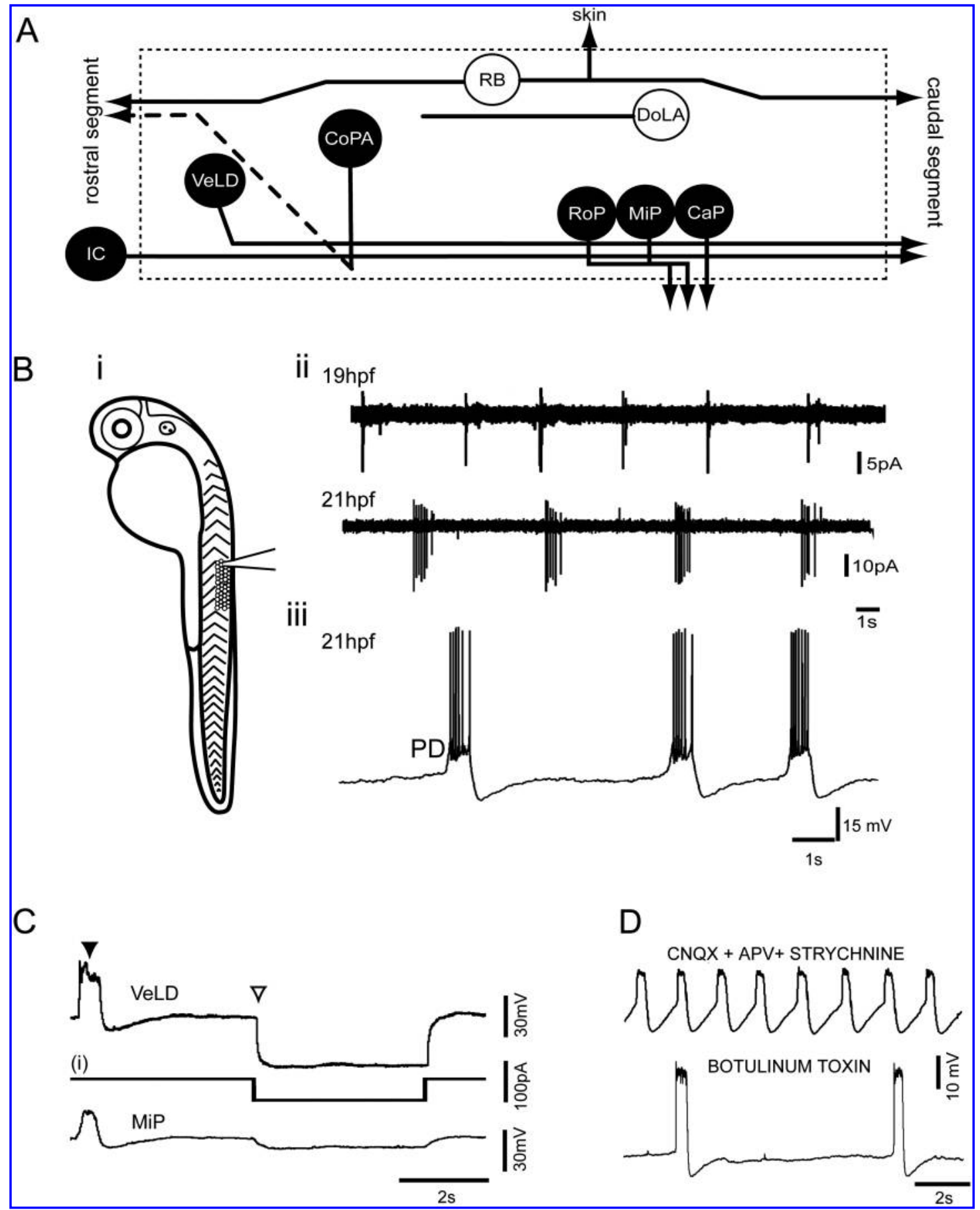

FIG. 1. Embryonic spinal cord. (A) Schematic diagram representing the spinal cord neurons that are present from 17 to $20 \mathrm{hpf}$. Black neurons are active during spontaneous activity, white neurons are silent. Dashed lines represent commissural projections. In this and other diagrams, dorsal is up, caudal is to the right. (Bi) Cartoon of a 1-day-old embryo showing the in vivo dissection and patch clamp approach; (Bii) cell attached traces from a primary motoneuron at 19 and $20 \mathrm{hpf}$ showing spontaneous repetitive bursting activity; (Biii) whole cell recording from a $21 \mathrm{hpf}$ primary motoneuron showing the periodic depolarizations (PD) that generate the spontaneous behavior. (C) Paired recording revealing synchronous activity between an interneuron and a motoneuron (black arrowhead) and the presence of strong electrical coupling (white arrowhead). (D) Pharmacology of the PDs, showing a lack of requirement for chemical neurotransmission. APV, CNQX, and strychnine are respectively NMDA, AMPA, and glycine receptor blockers. See Abbreviations for full chemical names. Figures modified from Refs. 38, 59, and 60. 
When observed under whole-cell configuration, all primary motoneurons show repetitive spontaneous periodic depolarizations (PD) (Fig. 1C). These PDs consist of sustained voltage increases lasting 300 to $500 \mathrm{~ms}$ upon which action potentials are often superimposed. Recordings from other spinal neurons further revealed that several interneurons also showed PDs. Indeed, PDs are observed in all IC and VeLD interneurons and in most of the CoPA interneurons from $19 \mathrm{hpf}$ to $24 \mathrm{hpf}$ (Fig. 1A, black neurons). Interestingly, Rohon-Beard and DoLA neurons never show PDs or spontaneous activity of any kind during the period of spontaneous activity in embryos. ${ }^{60}$ Paired simultaneous recordings from spinal neurons showed that PDs are synchronous in all active neurons and that these are electrically coupled to each other by gap junctions while inactive neurons are not coupled to any neurons ${ }^{59,60}$ (Fig. 1D). The gap junctions between spinal neurons were found to be necessary and sufficient for propagating the PDs.

Blocking chemical neurotransmission with cocktails of glutamatergic, GABAergic, and glycinergic receptor antagonists or blocking synaptic release with botulinum toxin does not abolish PDs (Fig. 1E), whereas procedures that block gap junctions extinguish the PDs. ${ }^{59}$ It is interesting to note that all neurons with PDs have axons that either course longitudinally in the VLF (IC, VeLD) or have axons that have ventral projections (CoPA), whereas inactive neurons (RB, DoLA) do not have any ventral projections. This observation suggests that either the active neurons contact each other at various points in the ventral spinal cord or that long descending axons link all the neurons together en passant. The finding that two $\mathrm{CaP}$ motoneurons in sequential somitic segments are electrically coupled to each other ${ }^{60}$ even though they have no possible direct point of contact strongly suggests that longitudinal axons can link other neurons to the electrotonic network all along the spinal cord. The connections are presumably axo-axonal or axo-somatic as the cell bodies of spinal neurons are devoid of dendrites at these early time points in development.

These results are consistent with gap junctions having a critical role in the propagation of PDs, but the mechanism by which PDs are initiated is still unknown. Tetrodotoxin (sodium channel blocker) blockade reveals that voltage dependent sodium channels are necessary for the generation of PDs, suggesting an important role for sodium influx. ${ }^{60}$ The increase of PD duration during apamin (Kca channel blocker) application further suggests that that there is significant calcium entry during PDs, and that calcium-activated potassium channels may play a role in PD termination. ${ }^{60}$ It will be interesting to determine whether the zebrafish rhythm originates from a pacemaker core as is the case for the lobster stomatogastric ganglion, where a few neurons have inherent pacemaker properties and drive the whole network ${ }^{61}$ or whether the zebrafish spinal cord pacemaker is more distributed and requires the active contribution of many different cell types and multiple electrical synaptic connections.

The presence of a pure electrotonic network in a developing vertebrate may seem unusual but there is a growing body of evidence that gap junctions play an important part in synchronizing early rhythms in the developing nervous system. During early development of the eye in mammals, waves of depolarizations spread across the retina. ${ }^{3}$ Dye-coupling experiments showed that gap junctions are present within retinal ganglion cells and amacrine cell populations, and the use of gap junction blockers demonstrated that retinal waves require gap junctions to propagate at very early stages. ${ }^{62}$ Disruption of retinal waves adversely affects axonal projections to the superior colliculus and eye-specific segregation of retinogeniculate projections. ${ }^{63}$ Interestingly, when chemical neurotransmission is perturbed at later stages when gap junctions are not normally required, gap junctions seem to upregulate and are able propagate the retinal waves. ${ }^{64}$ The leech has been shown to have extensive gap junctional coupling between neurons implicated in the production of motor activity. ${ }^{65-69}$

Gap junction-mediated networks also seem to play a role in the formation of the motor networks. In the developing chick spinal cord, gap junction blockers such as octanol and carbenoxolone were shown to reversibly abolish 
spontaneous bursting activity. ${ }^{70}$ Drug applications that alter the pattern of this bursting activity in the chick embryo have recently been shown to impair motor axon outgrowth to appropriate muscle targets. ${ }^{18}$ In addition, extensive dye junctional coupling in the spinal cord was recently observed at a very early stage in the embryonic mouse, ${ }^{71}$ and it is thought that the synchronization provided by this extensive coupling is permissive for the innervation of muscle by multiple motor axons during embryogenesis. After one week of postnatal life, as the electrical coupling diminishes, the synchronicity is lost and the normal course of synapse elimination is enabled. ${ }^{72}$ These results in many vertebrate animal models all point to a central importance of gap junction mediated synchronicity for the correct development of neuronal networks.

The appearance of the gap junction network in embryonic zebrafish may be a prerequisite for the formation of excitatory connections. The widespread electrical activity provided by the gap junction network may shape the formation of spinal circuitry. One of the roles of this network may be to synchronize the neurons and provide an electrical environment required for chemical synapse formation. It should be noted that experiments in which electrical activity is reduced during embryonic development ${ }^{49}$ support a significant role for activity-independent mechanisms in spinal circuit development, making the contribution of spontaneous activity unclear. Alternatively, the role of the gap junctions may not necessarily be to spread electrical activity but rather to connect prospective synaptic partners metabolically and enable the rapid spread of cellular messengers. Future experiments in which spontaneous activity is modified globally or in subsets of neurons while simultaneously monitoring efficacy at identified synapses are needed to test for the role of spontaneous activity in circuit formation.

\section{TACTILE RESPONSES AND CHEMICAL NEUROTRANSMISSION}

The next step in the progression of motor behaviors in the zebrafish embryo is the appear- ance of the touch response at $21 \mathrm{hpf}$. This new behavior suggests a change in the early gap junction-mediated motor network and implies at the very least, new functional connections with the sensory system. At this early time point, analysis of the touch response is complicated by the high frequency of spontaneous coiling. ${ }^{49}$ But by $23 \mathrm{hpf}$, the frequency of spontaneous coiling is sufficiently low to enable a detailed observation of the touch response. At this stage, head and tail stimulation produces the same response, which typically consists of a strong flexion of the trunk on the contralateral side, followed by one or two weaker alternating contractions. The initial contractions in response to touch are always stronger than the spontaneously occurring coils, suggesting a stronger recruitment of the musculature during touch responses. Although the isolated trunk responded to touch, the full flexure and strength are only seen when the hindbrain is intact. Brain structure above the hindbrain are not necessary for touch responses at $24 \mathrm{hpf}^{49}$ Touch responses are completely abolished when blockers of glutamatergic transmission are injected into the embryos (Fig. 2Bi), whereas spontaneous coiling continues at a normal frequency in the presence of the blockers (Fig. 2Bii). These behavioral results clearly show that hindbrain projections and chemical neurotransmission are already present by $21 \mathrm{hpf}$ in the zebrafish embryo and are required for normal touch responses.

The morphology of the spinal cord is changing rapidly at the stage when touch responses first appear. After $21 \mathrm{hpf}$, at least four more types of interneurons are added the spinal cord $^{51-53}$ (see gray neurons in Fig. 2A). Commissural secondary ascending (CoSA) interneurons project their axons like the CoPAs, first ventrally, then contralaterally and rostrally, but have smaller somata and extend their growth cones slightly later than CoPAs. Circumferential descending (CiD) interneurons send their axons first ventrally then caudally. Circumferential ascending (CiA) interneurons project ventrally to the floor plate, then rostrally on the same side of the embryo. Commissural bifurcating $(\mathrm{CoB})$ project ventrally, cross the midline, and then project axons both rostrally and caudally. Of these four later ar- 


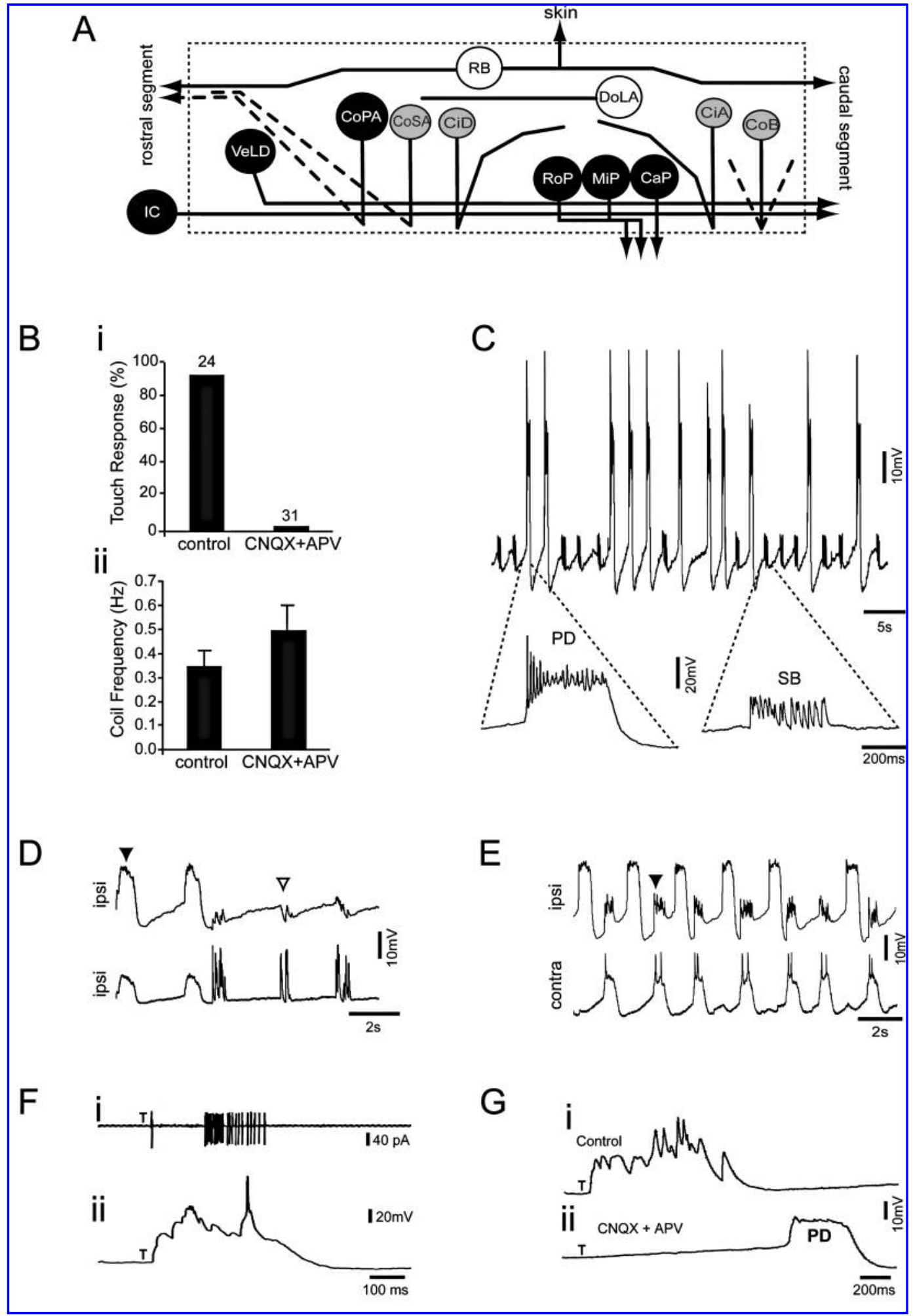

FIG. 2. Development of chemical synapses. (A) Schematic diagram representing the spinal cord neurons that are present from 21 to $26 \mathrm{hpf}$. (Bi) Block of the touch response by glutamatergic receptor blockers; (Bii) spontaneous contractions are unaffected by blocking glutamatergic transmission. (C) Whole cell patch clamp recording showing the appearance of bursts of glycinergic activity at $20 \mathrm{hpf}$ (SB). (D) Just as PDs (black arrowheads), SBs are synchronous in ipsilateral neurons (white arrowhead). (E) SBs are coincident with contralateral PDs. (F) Touch response recorded from a motoneuron in the cell-attached (i) and whole cell configuration (ii). (G) Touch responses are blocked by glutamatergic blockers (i) but PDs are unaffected (ii). See Abbreviations for full names. Figures modified from Refs. 38 and 60 . 
riving neurons, CoSAs are the only ones that reliably cross more than one somitic boundary before $24 \mathrm{hpf}$. A significant minority (45\%) of these later arriving neurons showed PDs $(23 / 51)$, suggesting that these neurons quickly wire into the existing gap junction network, presumably through their ventral projections.

Whole cell patch clamp from embryos after the onset of touch responses show that over $80 \%$ of primary motor neurons (PMN) display a new type of spontaneous rhythmic event in addition to the PDs that are already present. These events were named synaptic bursts (SB) because they are composed of rapid depolarizations that quickly return to baseline as opposed to the sustained drive during PDs (Fig. 2C). Synaptic bursts are present in most spinal neurons at the same frequency as the PDs but are sensitive to strychnine, a blocker of glycinergic transmission. ${ }^{59}$ Paired recordings revealed that SBs are simultaneous in pairs of ipsilateral spinal neurons (Fig. 2D), but are coincident with PDs on the contralateral side of the spinal cord (Fig. 2E). Due to high intracellular chloride, the glycine mediated events have been shown to be depolarizing at these early stages, ${ }^{59,73}$ but they are nevertheless thought to have an inhibitory shunting effect in motor networks as application of strychnine causes an abnormal synchronous activation of both sides of the animal. ${ }^{74}$

These results suggest that SBs represent commissural inhibition during contralateral excitation, akin to the midcycle inhibition seen during swimming in Xenopus larvae, which is required to prevent coactivation of both sides of the axial musculature during swimming. ${ }^{75}$ In Xenopus larvae, the glycinergic neurons responsible for the midcycle inhibition are the commissural interneurons, which project a commissural axon which bifurcates and extends both rostrally and caudally upon crossing the spinal cord. ${ }^{76}$ In the zebrafish, there are only three different types of commissural neurons at this stage: the CoPAs, CoSAs, and CoBs. The CoPA interneurons and a subset of CoSA interneurons were found to be positive for the presence of vesicular glutamate transporter mRNA at $32 \mathrm{hpf}$, indicating that these neurons are presumably glutamatergic interneurons ${ }^{77}$ and therefore not causing the SBs. The CoB in- terneurons and a second subset of CoSA interneurons on the other hand were found positive for mRNA encoding the neuronal glycine transporter, ${ }^{78}$ suggesting that they are good candidates for the commissural inhibitory interneurons responsible for the SBs.

When a day-old embryo is touched while performing a cell attached recording from an ipsilateral motoneuron, a single spike is observed, followed by a long pause of 150-200 ms and a spiking burst of 200-300 ms (Fig. 2Fi). This pattern is consistent with the behavior where the first contraction is always on the side opposite to the touch (Fig. 2Fi). When recording in the whole-cell configuration, touching the animal gives rise to a long summated burst of synaptic potentials that is longer in duration and larger in amplitude than the PDs (Fig. 2Fii). This touch response presumably propagates through the gap junctions in addition to using a mix of chemical synapses as it is hard to imagine gap junctions being selective for PDs but not to other electrical signals occurring at the same time.

The response to touch when recording from a VeLD interneuron is also composed of a similar burst of synaptic potentials, suggesting that the whole gap junction coupled ventral spinal cord is undergoing the same activity pattern upon touch (Fig. 2Gi). The burst of synaptic potentials in response to touch is completely absent when glutamate receptor blockers are added to the bath, but the PDs are still present (Fig. 2Gii). Because of limited knowledge of the wiring of the spinal cord in the zebrafish, it is still unclear where this stringent requirement for glutamatergic transmission comes from. The continued propagation of PDs through the gap junction network during the glutamatergic blockade of touch responses suggests that the sensory input never reaches the interconnected premotor network. The strict requirement for glutamate transmission in touch responses may come from the sensory to premotor neuron connections. More work is needed to address this possibility.

The appearance of responses to tail touch after $21 \mathrm{hpf}$ reveals that new connections are being made between sensory neurons and neurons in the motor network (Fig. 2F). The identification of the commissural CoPA in- 
terneurons as a glutamatergic interneuron puts this neuron at the head of a very short list to carry sensory excitation to the contralateral side of the spinal cord. ${ }^{77}$ Indeed, a very similar type of interneuron in the Xenopus larvae, the dorsolateral commissural sensory interneurons, is a glutamatergic interneuron responsible for relaying and amplifying sensory information from the Rohon-Beard sensory neurons to motoneurons and premotor interneurons on the opposite side of the spinal cord. ${ }^{79,80}$ It remains to be demonstrated by electrophysiological techniques whether this neuron is activated during embryonic touch responses and functions downstream of Rohon-Beard neurons.

Weaker contractions after lesions of the hindbrain suggest that reticulospinal projections are required to obtain the full strength touch response at $24 \mathrm{hpf}$, but it is still unclear which hindbrain neurons participate in the touch responses at this stage. The Mauthner neuron is presumably involved in the first manifestations of touch responses as its axon is already at the third spinal segment by 21 hpf. ${ }^{54,55}$ We can nevertheless hypothesize that the hindbrain neurons that get added to this early spinal circuit for the emergence of early touch responses continue to be involved in touch response with further development and may mediate the fast larval predator escape responses that can be seen after hatching. ${ }^{81}$

\section{LARVAL SPINAL CORD, ESCAPE, AND SWIMMING}

Embryonic zebrafish can be induced to swim as early as $28 \mathrm{hpf}$. The cycle (two alternating tail beats) frequency of the first observed swimming bouts is $8 \mathrm{~Hz}$ and the duration of the episodes is short lived. Slightly later in development, at $36 \mathrm{hpf}$, the embryos can swim at a $30 \mathrm{~Hz}$ cycle frequency. ${ }^{49}$ After normally hatching at 2 days, the larvae do not show much spontaneous motor activity, although swimming can normally be seen after a tactile stimulation. ${ }^{32,82}$ Two-day larvae swim in long uninterrupted bursts with tail beats that can reach a frequency of over $100 \mathrm{~Hz}$ and last tens of seconds. ${ }^{32,82}$ After 3 days of development, swimming switches to an intermittent mode of swimming. These motor events are composed of a string of a few cycles of tail beats punctuated by periods of inactivity. ${ }^{32,33,82}$ The level of spontaneous activity can be modulated; as recent research has shown that serotonin can modulate the level of motor activity in 3- to 4day-old larvae by changing chloride homeostasis and therefore cell excitability in spinal neurons. ${ }^{73,83}$

After 4 days, larvae have been shown to exhibit four main types of motor behaviors. Two of these motor gaits were classified as turning behaviors, namely, routine turns and escape turns. Routine turns are slower and weaker than escape turns and occur mostly spontaneously whereas escape turns are usually triggered by tactile stimulation and are an integral part of the startle response. ${ }^{84}$ The startle response is a predator escape motor behavior that has been very well studied in fishes, including the larval zebrafish. ${ }^{85,86}$ The startle response in larval zebrafish usually consists of a pronounced contralateral bend of the tail, the Cstart, which is usually followed by a weaker counter bend and an episode of swimming. ${ }^{81,84,87}$ The other two motor gaits seen at 4 days were classified as swimming behaviors and have been designated slow swims and burst swims. Slow swims typically follow routine turns whereas burst swims are associated to tactile evoked escape responses. Slow swim episodes involve weaker bends of the tail, lower frequencies of tail beats and slower swimming speeds than burst swimming. ${ }^{84}$

This simultaneous presence of weaker and stronger motor behaviors in zebrafish larvae suggests some flexibility in the recruitment of muscle fibers during behaviors. Indeed, muscle recordings in 3-day-old zebrafish larvae have shown that while embryonic red and white muscle cells usually received the same input, embryonic red muscle could be recruited independently of embryonic white muscle during slow swim episodes. Additionally, input to embryonic red muscle was shown to be attenuated during burst swimming episodes. ${ }^{33}$ This difference between slow and burst swimming is further highlighted by the observation that pectoral fins are used during slow swims but are tucked away during burst swimming. ${ }^{88}$ 
This differential recruitment of musculature suggests that that the motor circuitry may be composed of distinct subcircuits, or central pattern generators (CPG), that can act independently or in concert to produce different behaviors. ${ }^{84}$ By 6 days of development, zebrafish larvae show a larger repertoire of motor behaviors that includes prey tracking ${ }^{89}$ and prey capture, ${ }^{90}$ suggesting involvement of higher brain structures. The variability in larval motor behaviors presumably comes from differences in the descending control provided by functional subsets of reticulospinal neurons. ${ }^{36}$ Motor behaviors such as slow swims or routine turns may thus represent simple behavioral "building blocks" with which more complex behaviors are built.

It should prove interesting to study the changes in the underlying neural circuits that are required to go from the limited and stereotyped embryonic motor behaviors to the more complex and variable larval behaviors. One of the main substrates for these changes is the spinal cord. By 5 days of development, the spinal cord of zebrafish has changed dramatically from day 1 (Fig. 3A). Neurons that had short local projections such as the $\mathrm{CiD}$ in the embryo, now project axons several somites away from their cell bodies and spinal neurons now have extensive dendritic arborizations. ${ }^{91}$ It also seems that some neurons may change or modify their axonal projections, CiDs for example now have an additional rostral going projection and VeLDs (MCoD) now project contralaterally. New neurons are also added to the spinal cord. The first type of neuron, the CoLA neuron, has long ipsilateral rostral and caudal dendrites and they project a long commissural ascending axon. The second type of neuron, the UCoD neuron, also has an extensive dendritic arbor but has a descending commissural axon. ${ }^{91}$

What is remarkable is that even though the spinal cord is more complex at this later stage, it is still possible to identify individual neurons. This ability enables researchers to map the role of identified spinal neurons in the production of different motor behavior. Indeed, in vivo imaging of calcium transients while monitoring behavior has shown that CiDs are active only during escape responses and not swim- ming, while conversely MCoDs are only active during swimming and not escape responses (Fig. 3B). ${ }^{92}$ This intriguing result clearly shows that spinal circuitry is not simply shared for all behaviors and suggests that there are functional subdivisions in the zebrafish spinal cord. These findings support the concept that the spinal cord is composed of distinct, but presumably overlapping, CPGs. The inclusion or exclusion of a type of spinal neuron in the execution of a behavior likely results from differences in synaptic connectivity between reticulospinal neurons and their spinal targets.

Fictive motor activity can be recorded in the spinalized larvae under the right conditions, namely tonic NMDA application, but the spinal network is normally driven by reticulospinal inputs originating from the hindbrain. $34,81,87,93$ An identified reticulospinal neuron, the Mauthner cell and its homologues, has been implicated in escape responses in a variety of teleosts as well as in zebrafish. ${ }^{86,94,95}$ Experiments in zebrafish are beginning to assign behavioral functions to individual hindbrain neurons. Calcium imaging of the Mauthner and serial homologues has shown that a tail touch elicits activity in the Mauthner only, whereas a head touch recruits the Mauthner cell and homologues MiD2 $\mathrm{cm}$ and MiD3cm. ${ }^{81}$ This result reinforced the concept that the differential recruitment of hindbrain neurons may be responsible for the difference in behavior observed when touching either the head or the tail. ${ }^{85}$ This concept of differential recruitment was tested by ablating these neurons from zebrafish larvae and then testing their escape responses. Ablating all three Mauthner homologs abolished the short latency, high amplitude escape response, whereas ablating only the Mauthner neuron affected solely the performance of the tail evoked response. These results support the idea that these three neurons are differentially recruited to produce the behaviors appropriate to sensory context. ${ }^{93}$

These results highlight the power of imaging neuronal activity to probe the role of neurons in the production of behavior. The information gained from optical experiments can in turn be used to design more targeted electrophysiological experiments aimed at answering questions of connectivity, direction, and timing of 


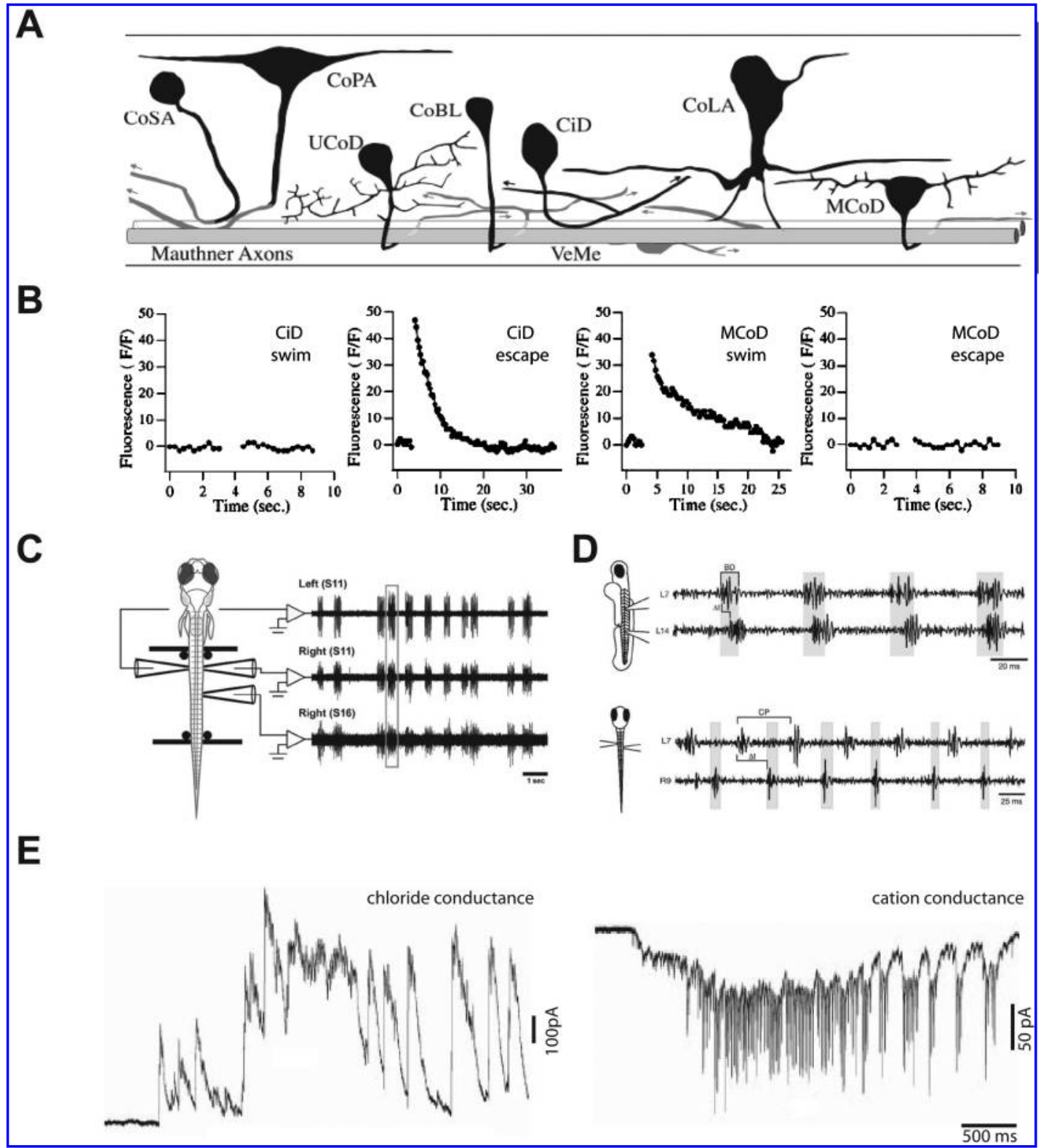

FIG. 3. The larval spinal cord. (A) Schematic diagram representing the spinal cord neurons that are present at 5 days. (B) Calcium imaging experiment showing that CiDs are only active during escape and $\mathrm{MCoD}$ are only active during swimming. (C) Schematic diagram of a ventral root recording technique and representative traces. (D) Ventral root recordings from spinalized larvae showing alternating activity. (E) Whole cell recordings from motoneuron during 2day-old burst swimming showing the isolation of the chloride conductance (i) and the cation conductance (ii). See Abbreviations for full names. Figures modified from Refs. 91 (A), 92 (B), 40 (C), 39 (D), and 32 (E) with permission.

information flow. Indeed, the combination of ventral root recordings and intracellular recordings from identified neurons has been used for several decades in the Xenopus and lamprey in order to map the circuitry responsible for swimming. These animal models have enabled some understanding of the cellular mechanisms underlying swimming in vertebrates but the precise cellular and ionic mech- anisms of rhythm generation are still elusive. $20,21,23,80,96,97$

Recently, techniques have been developed to record from the peripheral nerves of larval zebrafish during fictive swimming in intact larvae $^{40}$ and in spinalized larvae ${ }^{39}$ (Figs. 3C and 3D). These techniques will provide information about the phase relationships of the two sides of the spinal cord, information which is useful 
when trying to relate the activity from individual neurons to the rest of the network. In addition, ventral root recordings from spinalized zebrafish during NMDA-induced fictive swimming have provided evidence that very few spinal segments are necessary for oscillating activity. Indeed, lesions have shown that only two spinal segments are necessary for generating NMDA-induced rhythmic activity, ${ }^{39}$ which is consistent with results in other swimming vertebrates. ${ }^{98}$ Additional techniques have been developed to record from muscle cells ${ }^{33,99}$ and motoneurons. ${ }^{32,100}$ These techniques have begun to reveal the synaptic drive to motoneurons during fictive swimming as well as a complex pattern of motoneuron recruitment at early larval stages. Indeed, whole cell recordings from intact and spinalized larvae has shown that glycinergic drive has a tonic component, while the glutamatergic drive seems to have a stronger phasic component ${ }^{32,39}$ (Fig. 3E). The combination of these whole cell recordings from spinal motoneurons and interneurons with simultaneous ventral root recordings should enable researchers to identify the neurons that form the CPGs for simple motor behaviors in zebrafish. As we learn more about the networks that mediate the simpler sensory evoked motors behaviors we will be in a better position to understand how these hindbrain and spinal cord circuits are recruited by higher brain centers during more complex volitional behaviors.

\section{MOTILITY MUTANTS}

One of the great strengths of the zebrafish as an animal model is the ability to perform forward mutagenesis screens. One such mutagenesis screen performed in the Nüsslein-Volhard laboratory has generated over 30 distinct motility mutants with no visible muscle abnormality. ${ }^{26}$ Studying the genetic roots of these motor mutants should yield insights into motor circuit function in normal animals. ${ }^{24,101} \mathrm{Sev}$ eral of the motility mutants that have been studied to date affect motor activity by perturbing motor axon guidance, ${ }^{102-105}$ the neuromuscular junction, ${ }^{104,106-112}$ or excitation contraction coupling. ${ }^{111,113-116}$ A few motor mutants have been studied in more detail and have been shown to directly affect the central nervous system and will be described here.

Six mutants isolated by the Nüsslein-Volhard laboratory showed a decreased sensitivity to touch, yet were able to swim spontaneously. ${ }^{26}$ Three of these: macho, steifftier, and alligator, were selected for electrophysiological analysis because of the appearance of phenotypes early in development. All of these mutants were shown to have reduced voltage-dependent sodium currents in Rohon-Beard sensory neurons, which lead to the absence of overshooting action potentials. ${ }^{29}$ This inability of sensory cells to spike in the mutants is presumably the cause of their lack of mechanosensation. None of the genes for these mutations has yet been identified, yet the characterization of the mutant macho has already provided insights into an activity dependent developmental mechanism. Rohon-Beard neurons undergo programmed cell death in zebrafish and are completely eliminated from the spinal cord by 5 days postfertilization (dpf). ${ }^{117,118}$ The decreased activity in Rohon-Beard neurons of macho mutants seems to protect them from programmed cell death as they were shown to have less TUNEL positive staining and survive longer in macho mutants than in wild-type siblings. ${ }^{119}$

The deadly seven mutant was identified because of a somitogenesis defect, in which somite formation is disrupted after the sixth somite. ${ }^{120}$ Although this mutant was not identified as a motility mutant per se, it does cause the appearance of supernumerary neurons including multiple copies of the Mauthner cells. ${ }^{121}$ Kinematic analysis of the escape response in deadly seven mutants revealed little difference with wild-type siblings, suggesting that the extra Mauthner cells are either silent or integrate smoothly into the circuitry. ${ }^{122}$ Further results showed that all supernumerary Mauthners are active during touch, but each of them makes fewer synaptic contacts with neurons in the spinal cord, enabling the mutants to have normal escape responses despite the extra copies of Mauthner neurons. ${ }^{122}$ These results are interesting because they suggest a very high degree of plasticity in the development of the circuitry responsible for the escape response. 
The space cadet mutant is part of the twitch twice category of motor defects because they respond to tactile stimulation at $96 \mathrm{hpf}$ with multiple C-bends toward the same side instead of the normal touch response of a C-bend followed by a counter bend and swimming. ${ }^{26}$ The large magnitude of the successive $\mathrm{C}$-bends in the mutants suggested that the problem arose in the selection of the proper motor response and not the execution of the escape response itself, which pointed to a problem in the network of the Mauthner cell.123 Indeed, analysis of hindbrain morphology revealed a very specific defect of axonal projection in space cadet mutants, including the complete absence of the spiral fiber projections through the third hindbrain commissure. Moreover, lesioning of the same commissures in wild-type zebrafish resulted in the same aberrant phenotype. ${ }^{123}$ These missing projections normally give rise to the axon cap, which is a structure that has previously been shown to have a role in regulating the excitability of Mauthner cells in the goldfish. ${ }^{124-126}$ Although the search for the space cadet gene is still underway, this mutant has already provided novel insights into the structure and function of hindbrain motor network by showing a clear role for the spiral fibers in the production of a specific motor behavior. When identified, this gene will undoubtedly provide additional insights into axonal pathfinding mechanisms and the wiring of motor networks.

The mutant bandoneon was placed in the accordion group of mutations, because tactile stimulation causes both sides of the mutant to briefly contract simultaneously which reduces the length of the trunk much like an accordion. The spontaneous contractions occur normally in bandoneon mutants, but the responses to touch in the same animals at $24 \mathrm{~h}$ are clearly of the accordion type. ${ }^{74}$ When wild-type $24 \mathrm{hpf}$ embryos are placed in the glycine receptor blocker strychnine, they exhibit the same phenotype of normal spontaneous contractions with abnormal bilateral touch responses. This result suggests that bandoneon affects glycinergic synaptic transmission. The bandoneon gene was found to encode for the â2 subunit of the glycine receptor ( $g l r b 2)$. The defect in bandoneon was shown to result from the complete absence of clustering of the á subunit of the glycine receptor at glycinergic synapses, leading to the absence of synaptic glycinergic currents. ${ }^{74}$ The hypercontractility phenotype observed in bandoneon animals is reminiscent of what is observed in herperrekplexia, a disease involving impaired glycinergic transmission, causing patients to overreact with rigidity to unexpected stimuli. This mutant clearly highlights the fact that zebrafish mutants have potential to become important models to study genes implicated in human neurological diseases.

The shocked mutants were first isolated because of a lack of swimming. ${ }^{26}$ Further analysis of the mutants revealed that they cease spontaneous contractions abruptly at $21 \mathrm{hpf}$ and fail to respond to tactile stimulation at 24 hpf: by $48 \mathrm{hpf}$, the larvae respond to touch with a vigorous contralateral contraction but fail to initiate swimming. ${ }^{127,128}$ By 4 days, the mutants seem to recover some motor activity and show what may be a compensatory increase in electrical coupling between muscle cells, ${ }^{129}$ yet they start to die prematurely after 6 days. The mutation that causes shocked was found to inactivate the CNS glycine transporter (glyt1). ${ }^{128}$ Glyt1 is expressed extensively by non-neuronal cells in the hindbrain and spinal cord of embryos and larvae. It is thought that the absence of the glycine transporter in shocked mutants leads to an aberrant accumulation of glycine levels in the CNS and the shunting of sustained activity. ${ }^{128}$ Indeed, exposing the animals to strychnine recovers normal spontaneous coils in the embryos and partial swimming at 2 days, while removing glycine from the hindbrain during electrophysiological recordings uncovered a normal fictive swimming rhythm.

The investment of time and energy in the mutagenesis studies undertaken over a decade ago is beginning to bear fruit. The search for the locus of each mutation will identify proteins that are essential for the production of motor activity. It is surprising that a gene expressed in a glial cell can have a profound effect on the production of motricity, yet the mutant shocked provided evidence that absence of a glial cell glycine transporter prevents proper swimming rhythms. It is interesting to note that bandoneon and shocked both cause abrupt termination of motor rhythms, yet they are dif- 
ferent sides of the same glycinergic coin. These mutations reveal that proper rhythm generation can only occur with the right dose of inhibition. The glycinergic drive to motoneurons was found to be more tonic than the glutamatergic drive. ${ }^{32}$ These mutations reveal not only that this tonic drive is important but also that the amplitude of this tonic drive may be very critical. More work will be needed to uncover at which synapses and at which time glycinergic input to the motor pattern generator is critical for the production of rhythmic motor activity. Mutated genes are becoming easier to find as genomic sequence information is constantly being added to the databases. In the next decades we should be able to build a library of zebrafish mutants that affects every step required for motor behavior in order to characterize its development: from sensory perception to muscle contraction, and everything in between.

\section{FUTURE RESEARCH}

We still know very little about the circuitry required for complex motor behaviors in vertebrates. As shown above, optical techniques that take advantage of the transparent zebrafish larvae can be used to identify populations of spinal cord and hindbrain neurons that are active during swimming. Identification of these active neurons should be followed by lesion experiments ascribing a behavioral function to each type of neuron and ultimately to a detailed electrophysiological analysis of the synaptic drive mediated by these neurons during motor behaviors. This type of research will lead to a greater understanding of how vertebrate circuits generate rhythmic activity and behavior.

How these circuits initially develop is also a critical issue in biological research. The molecular mechanisms that lead to the precise synaptic connectivity during development in the embryonic spinal cord are still largely unknown. A variety of genetic tools and techniques are available in the zebrafish such as transgenic lines, ${ }^{130,131}$ enhancer lines, ${ }^{132-135}$ targeted protein knockdown with morpholinos ${ }^{136}$ and motility mutants. ${ }^{26}$ The combination of these various tools will permit researchers to modify the spinal cord expression patterns of any protein of interest in vivo while assaying the effects on neuron morphology, synaptic junction formation, network activity, and behavior. An area of promising interest to study the mechanisms involved in synaptic specificity is to look at proteins involved in cell-cell interactions such as the cadherin family of proteins. ${ }^{137,138}$

\section{CONCLUSIONS}

We are still at the beginning of this journey to the understanding of the genetics behind motor network assembly and function. One of the important determinants of the success of this journey is the development of powerful animal models that can reveal new information and promote new questions about circuit formation and function. The zebrafish is one such model, aligning the power of forward genetics, antisense knockdown, in vivo imaging, in vivo electrophysiology, and behavioral analysis. Research in the zebrafish has already shown that the motor network starts with a very simple circuit and builds complexity by adding layers of organization. Each new layer increases the range of possible motor acts from purely reflexive to volitional behaviors. Studying the formation of these basic motor networks and their maturation in zebrafish should lead to a better understanding of how animals go, as Grillner put it, "from egg to action."139

\section{ACKNOWLEDGMENTS}

Thanks to Mr. S.M. Sprague, Dr. E.J. Crespi, Dr. R.R. Buss, Dr. P. Drapeau, and Dr. J.Y. Kuwada for comments on the manuscript.

\section{ABBREVIATIONS}

APV, DL-2-Amino-5-phosphonovaleric acid; $\mathrm{CaP}$, caudal primary motoneuron; $\mathrm{CiA}$, circumferential ascending; CiD, circumferential descending; $\mathrm{CoB}$, commissural bifurcating; CNS, central nervous system; CNQX, 6-cyano7-nitroquinoxaline-2,3-dione; CoLA, commis- 
sural lateral ascending; CoPA, commissural primary ascending; CoSA, commissural secondary ascending; $\mathrm{CPG}$, central pattern generator; DLF, dorsal lateral fasciculus; DoLA, dorsal lateral ascending interneuron; glyR, glycine receptor; glyt1, glycine transporter 1; HPF, hours postfertilization; IC, interneuron with ipsilateral caudally directed axon; $\mathrm{MCoD}$, multipolar commissural descending; $\mathrm{MiP}$, middle primary motoneuron; NMDA, N-methyl-D-aspartate; PD, periodic depolarization; PMN, primary motoneuron; RB, Rohon-Beard sensory neuron; RoP, rostral primary motoneuron; SB, synaptic burst; UCoD, unipolar commissural descending; VeLD, ventral lateral descending interneuron; VLF, ventral lateral fasciculus.

\section{REFERENCES}

1. Ben-Ari Y. Developing networks play a similar melody. Trends Neurosci 2001;24:353-360.

2. Peinado A. Traveling slow waves of neural activity: a novel form of network activity in developing neocortex. J Neurosci 2000;20:RC54.

3. Feller MB. Spontaneous correlated activity in developing neural circuits. Neuron 1999;22:653-656.

4. Spitzer N.C. Coincidence detection enhances appropriate wiring of the nervous system. Proc Natl Acad Sci USA 2004;101:5311-5312.

5. Torborg CL, Feller MB. Spontaneous patterned retinal activity and the refinement of retinal projections. Prog Neurobiol 2005;76:213-235.

6. Torborg CL, Hansen KA, Feller MB. High frequency, synchronized bursting drives eye-specific segregation of retinogeniculate projections. Nat Neurosci 2005;8:72-78.

7. Spitzer NC. Activity-dependent neuronal differentiation prior to synapse formation: the functions of calcium transients. J Physiol Paris 2002;96:73-80.

8. Spitzer NC, Borodinsky LN, Root CM. Homeostatic activity-dependent paradigm for neurotransmitter specification. Cell Calcium 2005;37:417-423.

9. Borodinsky LN, Root CM, Cronin JA, Sann SB, Gu $X$, Spitzer NC. Activity-dependent homeostatic specification of transmitter expression in embryonic neurons. Nature 2004;429:523-530.

10. Hamburger V. Some aspects of the embryology of behavior. Q Rev Biol 1963;38:342-365.

11. Narayanan CH, Fox MW, Hamburger V. Prenatal development of spontaneous and evoked activity in the rat (Rattus norvegicus albinus). Behaviour 1971;40: 100-134.

12. Corner M. Spontaneous motor rhythms in early life-phenomenological and neurophysiological aspects. Prog Brain Res 1978;48:349-366.
13. Kudo N, Nishimaru H. Reorganization of locomotor activity during development in the prenatal rat. Ann NY Acad Sci 1998;860:306-317.

14. O'Donovan MJ, Wenner P, Chub N, Tabak J, Rinzel J. Mechanisms of spontaneous activity in the developing spinal cord and their relevance to locomotion. Ann NY Acad Sci 1998;860:130-141.

15. Ren J, Greer JJ. Ontogeny of rhythmic motor patterns generated in the embryonic rat spinal cord. $\underline{\mathrm{Neu}}$ rophysiol 2003;89:1187-1195.

16. Yvert B, Branchereau P, Meyrand P. Multiple spontaneous rhythmic activity patterns generated by the embryonic mouse spinal cord occur within a specific developmental time window. J Neurophysiol 2004; 91:2101-2109.

17. O'Donovan MJ, Bonnot A, Wenner P, Mentis GZ. Calcium imaging of network function in the developing spinal cord. Cell Calcium 2005;37:443-450.

18. Hanson MG, Landmesser LT. Normal patterns of spontaneous activity are required for correct motor axon guidance and the expression of specific guidance molecules. Neuron 2004;43:687-701.

19. Myers CP, Lewcock JW, Hanson MG, Gosgnach S, Aimone JB, Gage FH, et al. Cholinergic input is required during embryonic development to mediate proper assembly of spinal locomotor circuits. Neuron 2005;46:37-49.

20. Roberts A. How does a nervous system produce behaviour? A case study in neurobiology. Sci Prog 1990;74:31-51.

21. Roberts A, Soffe SR, Wolf ES, Yoshida M, Zhao FY. Central circuits controlling locomotion in young frog tadpoles. Ann NY Acad Sci 1998;860:19-34.

22. Grillner S. The motor infrastructure: from ion channels to neuronal networks. Nat Rev Neurosci 2003; 4:573-586.

23. Grillner S, Kozlov A, Kotaleski JH. Integrative neuroscience: linking levels of analyses. Curr Opin Neurobiol 2005;15:614-621.

24. Fetcho JR, Liu KS. Zebrafish as a model system for studying neuronal circuits and behavior. Ann NY Acad Sci 1998;860:333-345.

25. Kullander K. Genetics moving to neuronal networks. Trends Neurosci 2005;28:239-247.

26. Granato M, van Eeden FJ, Schach U, Trowe T, Brand M, Furutani-Seiki $M$, et al. Genes controlling and mediating locomotion behavior of the zebrafish embryo and larva. Development 1996;123:399-413.

27. Golling G, Amsterdam A, Sun Z, Antonelli M, Maldonado $\mathrm{E}, \mathrm{Chen} \mathrm{W}$, et al. Insertional mutagenesis in zebrafish rapidly identifies genes essential for early vertebrate development. Nat Genet 2002;31: 135-140.

28. Fetcho JR, O'Malley DM. Imaging neuronal networks in behaving animals. Curr Opin Neurobiol 1997;7:832-838.

29. Ribera AB, Nusslein-Volhard C. Zebrafish touch-insensitive mutants reveal an essential role for the developmental regulation of sodium current. I Neurosci 1998;18:9181-9191. 
30. Drapeau P, Ali DW, Buss RR, Saint-Amant L. In vivo recording from identifiable neurons of the locomotor network in the developing zebrafish. J Neurosci Meth 1999;88:1-13.

31. Ali DW, Buss RR, Drapeau P. Properties of miniature glutamatergic EPSCs in neurons of the locomotor regions of the developing zebrafish. I Neurophysiol 2000;83:181-191.

32. Buss RR, Drapeau P. Synaptic drive to motoneurons during fictive swimming in the developing zebrafish. J Neurophysiol 2001;86:197-210.

33. Buss RR, Drapeau P. Activation of embryonic red and white muscle fibers during fictive swimming in the developing zebrafish. J Neurophysiol 2002;87: 1244-1251.

34. Takahashi M, Narushima M, Oda Y. In vivo imaging of functional inhibitory networks on the mauthner cell of larval zebrafish. J Neurosci 2002;22: 3929-3938.

35. Higashijima S, Masino MA, Mandel G, Fetcho JR. Imaging neuronal activity during zebrafish behavior with a genetically encoded calcium indicator. I Neurophysiol 2003;90:3986-3997.

36. O'Malley DM, Zhou Q, Gahtan E. Probing neural circuits in the zebrafish: a suite of optical techniques. Methods 2003;30:49-63.

37. Brustein E, Marandi N, Kovalchuk Y, Drapeau P, Konnerth A. "In vivo" monitoring of neuronal network activity in zebrafish by two-photon $\mathrm{Ca}(2+)$ imaging. Pflugers Arch 2003;446:766-773.

38. Saint-Amant L, Drapeau P. Whole-cell patch-clamp recordings from identified spinal neurons in the zebrafish embryo. Methods Cell Sci 2003;25:59-64.

39. McDearmid JR, Drapeau P. Rhythmic motor activity evoked by NMDA in the spinal zebrafish larva. I Neurophysiol 2006;95:401-417.

40. Masino MA, Fetcho JR. Fictive swimming motor patterns in wild type and mutant larval zebrafish. I Neurophysiol 2005;93:3177-3188.

41. Appel B. Zebrafish neural induction and patterning. Dev Dyn 2000;219:155-168.

42. Lewis KE, Eisen JS. From cells to circuits: development of the zebrafish spinal cord. Prog Neurobiol 2003;69:419-449.

43. Beattie CE. Control of motor axon guidance in the zebrafish embryo. Brain Res Bull 2000;53:489-500.

44. Beattie CE, Granato M, Kuwada JY. Cellular, genetic and molecular mechanisms of axonal guidance in the zebrafish. Results Probl Cell Differ 2002;40:252-269.

45. Hjorth J, Key B. Development of axon pathways in the zebrafish central nervous system. Int J Dev Biol 2002;46:609-619.

46. Drapeau P, Saint-Amant L, Buss RR, Chong M, McDearmid JR, Brustein E. Development of the locomotor network in zebrafish. Prog Neurobiol 2002;68:85-111.

47. Brustein E, Saint-Amant L, Buss RR, Chong M, McDearmid JR, Drapeau P. Steps during the development of the zebrafish locomotor network. J Physiol Paris 2003;97:77-86.
48. Kimmel CB, Patterson J, Kimmel RO. The development and behavioral characteristics of the startle response in the zebra fish. Dev Psychobiol 1974;7: $47-60$.

49. Saint-Amant L, Drapeau P. Time course of the development of motor behaviors in the zebrafish embryo. I Neurobiol 1998;37:622-632.

50. Grunwald DJ, Kimmel CB, Westerfield M, Walker C, Streisinger G. A neural degeneration mutation that spares primary neurons in the zebrafish. Dev Biol 1988;126:115-128.

51. Bernhardt RR, Chitnis AB, Lindamer L, Kuwada JY. Identification of spinal neurons in the embryonic and larval zebrafish. J Comp Neurol 1990;302: 603-616.

52. Kuwada JY, Bernhardt RR. Axonal outgrowth by identified neurons in the spinal cord of zebrafish embryos. Exp Neurol 1990;109:29-34.

53. Kuwada JY, Bernhardt RR, Nguyen N. Development of spinal neurons and tracts in the zebrafish embryo. J Comp Neurol 1990;302:617-628.

54. Mendelson B. Development of reticulospinal neurons of the zebrafish. I. Time of origin. J Comp Neurol 1986;251:160-171.

55. Mendelson B. Development of reticulospinal neurons of the zebrafish. II. Early axonal outgrowth and cell body position. J Comp Neurol 1986;251:172-184.

56. Myers PZ. Spinal motoneurons of the larval zebrafish. J Comp Neurol 1985;236:555-561.

57. Eisen JS, Myers PZ, Westerfield M. Pathway selection by growth cones of identified motoneurones in live zebra fish embryos. Nature 1986;320:269-271.

58. Westerfield M, McMurray JV, Eisen JS. Identified motoneurons and their innervation of axial muscles in the zebrafish. J Neurosci 1986;6:2267-2277.

59. Saint-Amant L, Drapeau P. Motoneuron activity patterns related to the earliest behavior of the zebrafish embryo. I Neurosci 2000;20:3964-3972.

60. Saint-Amant L, Drapeau P. Synchronization of an embryonic network of identified spinal interneurons solely by electrical coupling. Neuron 2001;31: 1035-1046.

61. Marder E. Roles for electrical coupling in neural circuits as revealed by selective neuronal deletions. I Exp Biol 1984;112:147-167.

62. Syed MM, Lee S, Zheng J, Zhou ZJ. Stage-dependent dynamics and modulation of spontaneous waves in the developing rabbit retina. I Physiol 2004;560: 533-549.

63. Firth SI, Wang CT, Feller MB. Retinal waves: mechanisms and function in visual system development. Cell Calcium 2005;37:425-432.

64. Stacy RC, Demas J, Burgess RW, Sanes JR, Wong RO. Disruption and recovery of patterned retinal activity in the absence of acetylcholine. J Neurosci 2005; 25:9347-9357.

65. Wolszon LR, Rehder V, Kater SB, Macagno ER. Calcium wave fronts that cross gap junctions may signal neuronal death during development. J Neurosci 1994;14:3437-3448. 
66. Rela L, Szczupak L. Coactivation of motoneurons regulated by a network combining electrical and chemical synapses. J Neurosci 2003;23:682-692.

67. Fan RJ, Marin-Burgin A, French KA, Otto Friesen W. A dye mixture (Neurobiotin and Alexa 488) reveals extensive dye-coupling among neurons in leeches; physiology confirms the connections. I Comp Physiol A Neuroethol Sens Neural Behav Physiol 2005;191:1157-1171.

68. Marin-Burgin A, Eisenhart FJ, Baca SM, Kristan WB Jr, French KA. Sequential development of electrical and chemical synaptic connections generates a specific behavioral circuit in the leech. J Neurosci 2005;25:2478-2489.

69. Marin-Burgin A, Eisenhart FJ, Kristan WB Jr, French KA. Embryonic electrical connections appear to prefigure a behavioral circuit in the leech CNS. I Comp Physiol A Neuroethol Sens Neural Behav Physiol, 2006;192:123-133.

70. Milner LD, Landmesser LT. Cholinergic and GABAergic inputs drive patterned spontaneous motoneuron activity before target contact. I Neurosci 1999;19:3007-3022.

71. Bittman KS, Panzer JA, Balice-Gordon RJ. Patterns of cell-cell coupling in embryonic spinal cord studied via ballistic delivery of gap-junction-permeable dyes. I Comp Neurol 2004;477:273-285.

72. Wyatt RM, Balice-Gordon RJ. Activity-dependent elimination of neuromuscular synapses. J Neurocytol 2003;32:777-794.

73. Brustein E, Drapeau P. Serotoninergic modulation of chloride homeostasis during maturation of the locomotor network in zebrafish. I Neurosci 2005;25: 10607-10616.

74. Hirata H. Saint-Amant L, Downes GB, Cui WW, Zhou W, Granato M, et al. Zebrafish bandoneon mutants display behavioral defects due to a mutation in the glycine receptor beta-subunit. Proc Natl Acad Sci USA 2005;102:8345-8350.

75. Soffe SR, Clarke JD, Roberts A. Activity of commissural interneurons in spinal cord of Xenopus embryos. I Neurophysiol 1984;51:1257-1267.

76. Li WC, Ferrins R, Soffe SR, Yoshida M, Walford A, Roberts A. Defining classes of spinal interneuron and their axonal projections in hatchling Xenopus laevis tadpoles. J Comp Neurol 2001;441:248-265.

77. Higashijima S, Mandel G, Fetcho JR. Distribution of prospective glutamatergic, glycinergic, and GABAergic neurons in embryonic and larval zebrafish. J Comp Neurol 2004;480:1-18.

78. Higashijima S, Schaefer M, Fetcho JR. Neurotransmitter properties of spinal interneurons in embryonic and larval zebrafish. J Comp Neurol 2004;480:19-37.

79. Li WC, Soffe SR, Roberts A. The spinal interneurons and properties of glutamatergic synapses in a primitive vertebrate cutaneous flexion reflex. J Neurosci 2003;23:9068-9077.

80. Roberts A. Early functional organization of spinal neurons in developing lower vertebrates. Brain Res Bull 2000;53:585-593.
81. O'Malley DM, Kao YH, Fetcho J. R. Imaging the functional organization of zebrafish hindbrain segments during escape behaviors. Neuron 1996;17: 1145-1155.

82. Muller UK, van Leeuwen JL. Swimming of larval zebrafish: ontogeny of body waves and implications for locomotory development. J Exp Biol 2004;207: 853-868.

83. Brustein E, Chong M, Holmqvist B, Drapeau P. Serotonin patterns locomotor network activity in the developing zebrafish by modulating quiescent periods. I Neurobiol 2003;57:303-322.

84. Budick SA, O'Malley DM. Locomotor repertoire of the larval zebrafish: swimming, turning and prey capture. J Exp Biol 2000;203:2565-2579.

85. Foreman MB, Eaton RC. The direction change concept for reticulospinal control of goldfish escape. I Neurosci 1993;13:4101-4113.

86. Eaton RC, Lee RK, Foreman MB. The Mauthner cell and other identified neurons of the brainstem escape network of fish. Prog Neurobiol 2001;63:467-485.

87. Gahtan E, Sankrithi N, Campos JB, O'Malley DM. Evidence for a widespread brain stem escape network in larval zebrafish. J Neurophysiol 2002:87: 608-614.

88. Thorsen DH, Cassidy JJ, Hale ME. Swimming of larval zebrafish: fin-axis coordination and implications for function and neural control. J Exp Biol 2004; 207:4175-4183.

89. McElligott MB, O'Malley DM. Prey tracking by larval zebrafish: axial kinematics and visual control. Brain Behav Evol 2005;66:177-196.

90. Borla MA, Palecek B, Budick S, O’Malley DM. Prey capture by larval zebrafish: evidence for fine axial motor control. Brain Behav Evol 2002;60:207-229.

91. Hale ME, Ritter DA, Fetcho JR. A confocal study of spinal interneurons in living larval zebrafish. $\underline{\text { IComp }}$ Neurol 2001;437:1-16.

92. Ritter DA, Bhatt DH, Fetcho JR. In vivo imaging of zebrafish reveals differences in the spinal networks for escape and swimming movements. J Neurosci 2001;21:8956-8965.

93. Liu KS, Fetcho JR. Laser ablations reveal functional relationships of segmental hindbrain neurons in zebrafish. Neuron 1999;2:325-335.

94. Eaton RC, Bombardieri RA, Meyer DL. The Mauthner-initiated startle response in teleost fish. J Exp Biol 1977;66:65-81.

95. Eaton RC, DiDomenico R, Nissanov J. Role of the Mauthner cell in sensorimotor integration by the brain stem escape network. Brain Behav Evol 1991;37:272-285.

96. Grillner S, Wallen P. On the cellular bases of vertebrate locomotion. Prog Brain Res 1999;123:297-309.

97. Grillner S, Cangiano L, Hu G, Thompson R, Hill R, Wallen $P$. The intrinsic function of a motor systemfrom ion channels to networks and behavior. Brain Res 2000;886:224-236.

98. Grillner S, Wallen P, Brodin L, Lansner A. Neuronal network generating locomotor behavior in lamprey: 
circuitry, transmitters, membrane properties, and simulation. Annu Rev Neurosci 1991;14:169-199.

99. Buss RR, Drapeau P. Physiological properties of zebrafish embryonic red and white muscle fibers during early development. I Neurophysiol 2000;84: 1545-1557.

100. Buss RR, Bourque CW, Drapeau P. Membrane properties related to the firing behavior of zebrafish motoneurons. J Neurophysiol 2003;89:657-664.

101. Bate M. Development of motor behaviour. Curr Opin Neurobiol 1999;9:670-675.

102. Zeller J, Granato M. The zebrafish diwanka gene controls an early step of motor growth cone migration. Development 1999;126:3461-3472.

103. Rodino-Klapac LR, Beattie CE. Zebrafish topped is required for ventral motor axon guidance. Dev Biol 2004;273:308-320.

104. Zhang J, Lefebvre JL, Zhao S, Granato M. Zebrafish unplugged reveals a role for muscle-specific kinase homologs in axonal pathway choice. Nat Neurosci 2004;7:1303-1309.

105. Birely J, Scheider VA, Santana E, Dosch R, Wagner DS, Mullino MC, et al. Genetic screens for genes controlling motor nerve-muscle development and interactions. Dev Biol 2005;280:162-176.

106. Panzer JA, Gibbs SM, Dosch R, Wagner D, Mullins MC, Granato M, et al. Neuromuscular synaptogenesis in wild-type and mutant zebrafish. Dev Biol 2005;285:340-357.

107. Lefebvre JL, Ono F, Puglielli C, Seidner G, FranziniArmstrong C, Brehm P, et al. Increased neuromuscular activity causes axonal defects and muscular degeneration. Development 2004;131:2605-2618.

108. Downes GB, Granato M. Acetylcholinesterase function is dispensable for sensory neurite growth but is critical for neuromuscular synapse stability. Dev Biol 2004;270:232-245.

109. Behra M, Cousin X, Bertrand C, Vonesch JL, Biellmann D, Chatonnet A, et al. Acetylcholinesterase is required for neuronal and muscular development in the zebrafish embryo. Nat Neurosci 2002;5:111-118.

110. Ono F, Shcherbatko A, Higashijima S, Mandel G, Brehm, P. The zebrafish motility mutant twitch once reveals new roles for rapsyn in synaptic function. I Neurosci 2002;22:6491-6498.

111. Ono F, Higashijima S, Shcherbatko A, Fetcho JR, Brehm P. Paralytic zebrafish lacking acetylcholine receptors fail to localize rapsyn clusters to the synapse. J Neurosci 2001;21:5439-5448.

112. Westerfield M, Liu DW, Kimmel CB, Walker C. Pathfinding and synapse formation in a zebrafish mutant lacking functional acetylcholine receptors. Neuron 1990;4:867-874.

113. Hirata H, Saint-Amant L, Waterbury J, Cui WW, Zhou W, Li Q, et al. accordion, a zebrafish behavioral mutant, has a muscle relaxation defect due to a mutation in the ATPase Ca2+ pump SERCA1. Development 2004;131:5457-5468.

114. Gleason MR, Armisen R, Verdecia MA, Sirotkin $H$, Brehm P, Mandel G. A mutation in serca underlies motility dysfunction in accordion zebrafish. Dev Biol 2004;276:441-451.

115. Schredelseker J, Di Biase V, Obermair GJ, Felder ET, Flucher BE, Franzini-Armstrong C, et al. The beta 1a subunit is essential for the assembly of dihydropyridine-receptor arrays in skeletal muscle. Proc Natl Acad Sci USA 2005;102:17219-17224.

116. Zhou W, Saint-Amant L, Hirata H, Cui WW, Sprague SM, Kuwada JY. Non-sense mutations in the dihydropyridine receptor beta1 gene, CACNB1, paralyze zebrafish relaxed mutants. Cell Calcium 2006;39: 227-236.

117. Williams JA, Barrios A, Gatchalian C, Rubin L, Wilson SW, Holder N. Programmed cell death in zebrafish rohon beard neurons is influenced by TrkC1/NT-3 signaling. Dev Biol 2000;226:220-230.

118. Reyes R, Haendel M, Grant D, Melancon E, Eisen JS. Slow degeneration of zebrafish Rohon-Beard neurons during programmed cell death. Dev Dyn 2004;229:30-41.

119. Svoboda KR, Linares AE, Ribera AB. Activity regulates programmed cell death of zebrafish Rohon-Beard neurons. Development 2001;128:3511-3520.

120. van Eeden FJ, Granato M, Schach U, Brand M, Furutani-Seiki $\mathrm{M}$, Haffter $\mathrm{P}$, et al. Mutations affecting somite formation and patterning in the zebrafish, Danio rerio. Development 1996;123:153-164.

121. Gray M, Moens CB, Amacher SL, Eisen JS, Beattie CE. Zebrafish deadly seven functions in neurogenesis. Dev Biol 2001;237:306-323.

122. Liu KS, Gray M, Otto SJ, Fetcho JR, Beattie CE. Mutations in deadly seven/notch1a reveal developmental plasticity in the escape response circuit. I Neurosci 2003;23:8159-8166.

123. Lorent K, Liu KS, Fetcho JR, Granato M. The zebrafish space cadet gene controls axonal pathfinding of neurons that modulate fast turning movements. Development 2001;128:2131-2142.

124. Furukawa T, Fukami Y, Asada Y. A third type of inhibition in the Mauthner cell of goldfish. I Neurophysiol 1963;26:759-774.

125. Furukawa T, Furshpan EJ. Two inhibitory mechanisms in the Mauthner neurons of goldfish. I Neurophysiol 1963;26:140-176.

126. Scott JW, Zottoli SJ, Beatty NP, Korn H. Origin and function of spiral fibers projecting to the goldfish Mauthner cell. J Comp Neurol 1994;339:76-90.

127. Cui WW, Saint-Amant L, Kuwada JY. shocked Gene is required for the function of a premotor network in the zebrafish CNS. J Neurophysiol 2004;92: 2898-2908.

128. Cui WW, Low SE, Hirata H, Saint-Amant L, Geisler $\mathrm{R}$, Hume RI, et al. The zebrafish shocked gene encodes a glycine transporter and is essential for the function of early neural circuits in the CNS. J Neurosci 2005;25:6610-6620.

129. Luna VM, Wang M, Ono F, Gleason MR, Dallman JE, Mandel G, et al. Persistent electrical coupling and locomotory dysfunction in the zebrafish mutant shocked. J Neurophysiol 2004;92:2003-2009. 
130. Kuwada JY. Development of the zebrafish nervous system: genetic analysis and manipulation. Curr Opin Neurobiol 1995;5:50-54.

131. Kawakami K. Transposon tools and methods in zebrafish. Dev Dyn 2005;234:244-254.

132. Balciunas D, Davidson AE, Sivasubbu S, Hermanson SB, Welle Z, Ekker SC. Enhancer trapping in zebrafish using the Sleeping Beauty transposon. BMC Genomics 2004;5:62.

133. Parinov S, Kondrichin I, Korzh V, Emelyanov A. Tol2 transposon-mediated enhancer trap to identify developmentally regulated zebrafish genes in vivo. Dev Dyn 2004;231:449-459.

134. Woolfe A, Goodson M, Goode DK, Snell P, McEwen GK, Vavouri T, et al. Highly conserved non-coding sequences are associated with vertebrate development. PLoS Biol 2005;3:e7.

135. Ellingsen S, Laplante MA, Konig M, Kikuta H, Furmanek T, Hoivik EA, et al. Large-scale enhancer detection in the zebrafish genome. Development 2005;132:3799-3811.
136. Nasevicius A, Ekker SC. Effective targeted gene 'knockdown' in zebrafish. Nat Genet 2000;26:216220.

137. Jontes JD, Emond MR, Smith SJ. In vivo trafficking and targeting of $\mathrm{N}$-cadherin to nascent presynaptic terminals. J Neurosci 2004;24:9027-9034.

138. Down M, Power M, Smith SI, Ralston K, Spanvello $\mathrm{M}$, Burno GF, et al. Cloning and expression of the large zebrafish protocadherin gene, Fat. Gene Expr Patterns 2005;5:483-490.

139. Grillner S. From egg to action. Brain Res Bull 2000;53:473-477.

Address reprint requests to: Louis Saint-Amant University of Michigan 815 North University Ann Arbor, MI 48109-1048

E-mail: louiss@umich.edu 
This article has been cited by:

1. 2006. Recent Papers on Zebrafish and Other Aquarium Fish ModelsRecent Papers on Zebrafish and Other Aquarium Fish Models. Zebrafish 3:3, 387-398. [Citation] [PDF] [PDF Plus] 